\title{
Prions hijack the nanotube
}

Researchers have long sought to understand how prions spread to and through the central nervous system. Reporting in Nature Cell Biology, Chiara Zurzolo and her colleagues now show for the first time that prions can spread from cell to cell using tunnelling nanotubes.

Previous studies have found that some pathogens, such as HIV, ride tunnelling nanotubes, thin projections that connect cells together. To determine whether prion spread involves the same system, the authors used fluorescent dyes to label nanotubes and prions in CAD cells, a mouse neuronal cell line, and observed wild-type prion protein travelling through the tunnelling nanotubes that connect cells. Similarly, fluorescently labelled $\mathrm{PrP}^{\mathrm{Sc}}$, the misfolded form of the prion protein that causes disease, also rides the tunnelling nanotube. Given the speed and pattern of prion migration, the authors suspect that prions travel by vesicular transport inside these tubes.

To confirm that $\mathrm{PrP}^{\mathrm{sc}}$ was spreading through tunnelling nanotubes, the authors next incubated $\mathrm{PrP}^{\mathrm{Sc}}$-infected CAD cells with non-infected CAD cells. After overnight co-incubations,

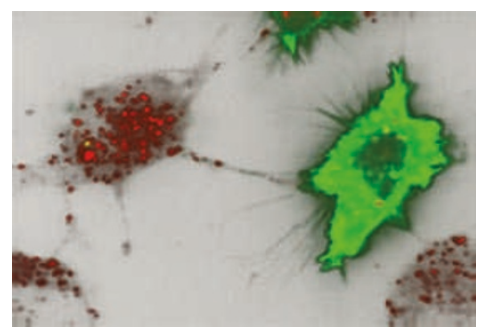

A tunnelling nanotube connects two CAD cells. Image courtesy of C. Zurzolo, The Pasteur Institute, France. the authors found that $\mathrm{PrP}^{\mathrm{Sc}}$ had spread from infected CAD cells to previously uninfected CAD cells, but only when they had been connected by naturally occurring nanotubes. If cells were in direct physical contact but were not joined by nanotubes, $\mathrm{PrP}^{\mathrm{Sc}}$ did not spread.

Finally, the authors examined whether tunnelling nanotubes might be important for prion spread between other cell types. They found that tunnelling nanotubes enabled efficient transfer of $\operatorname{Pr} \mathrm{P}^{\mathrm{Sc}}$ from dendritic cells to CAD cells and to primary neurons in overnight culture. Notably, researchers have previously proposed that dendritic cells might have an important role in spreading prions from the gut to the nervous system.

Taken together, these finding suggest that prions gain access into and between neurons by hijacking tunnelling nanotubes. Whereas previous studies have shown that prions can spread by other mechanisms, these are far less efficient. For example, transportation by exosomes requires 5 days of co-culture, compared with 12 hours by nanotubes.

Asher Mullard

ORIGINAL RESEARCH PAPER Gousset, K. et al. Prions hijack tunnelling nanotubes for intercellular spread. Nature Cell Biol. 8 Feb 2009 (doi: 10.1038/ncb1841)

FURTHER READING Davis, D. M. \& Sowinski, S. Membrane nanotubes: dynamic long-distance connections between animal cells. Nature Rev. Mol. Cell Biol. 9, 431-436 (2008) 